\title{
Large field-of-view asymmetric masks for high-energy X-ray phase imaging with standard X-ray tube
}

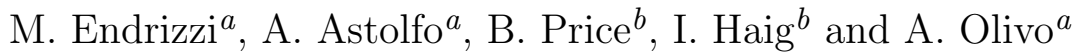 \\ ${ }^{a}$ Department of Medical Physics and Biomedical Engineering, University College London, \\ Gower Street, London WC1E 6BT, United Kingdom \\ ${ }^{b}$ Nikon Metrology UK Ltd., Unit 5, Icknield Industrial Estate, Tring, Herts HP23 4JX, United \\ Kingdom
}

\begin{abstract}
We report on a new approach to large field-of-view laboratory-based X-ray phase-contrast imaging. The method is based upon the asymmetric mask design that enables the retrieval of the absorption, refraction and ultra-smallangle scattering properties of the sample without the need to move any component of the imaging system. The sample is scanned through the imaging system, which also removes possible aliasing problems that might arise from partial sample illumination when using the edge illumination technique. This concept can be extended to any desired number of apertures providing, at the same time, intensity projections at complementary illumination conditions. Experimental data simultaneously acquired at seven different illumination fractions are presented along with the results obtained from a numerical model that incorporates the actual detector performance. The ultimate shape of the illumination function is shown to be significantly dependent on these detector-specific characteristics. Based on this concept, a large field-of-view system was designed, which is also capable to cope with relatively high $(100 \mathrm{kVp})$ X-ray energies. The imaging system obtained in this way, where the asymmetric mask design enables the data to be collected without moving any element of the instrumentation, adapts particularly well to those situations in medical, industrial and security imaging where the sample has to be scanned through the system.
\end{abstract}

Keywords: X-ray imaging, phase-contrast imaging, edge-illumination

\section{INTRODUCTION}

$\mathrm{X}$-ray imaging is an invaluable tool for the non destructive inspection of the internal structure of objects and finds application in a large variety of different fields, ranging from biological and medical sciences to materials engineering and security screening. In conventional radiography, contrast is solely generated by the differences in the attenuation that the X-rays encounter while passing through the sample under investigation. This capability can be extended by means of a phase-contrast imaging system, where also the phase shifts, imparted to the $\mathrm{X}$-ray wave that travels through the sample, contribute to the modulation of the intensity recorded by the image receptor. ${ }^{1-3}$ This approach bears great potential for the enhancement of image contrast, especially in those cases where intrinsically weakly absorbing samples are investigated.

X-ray phase-contrast imaging (XPCI) can be achieved, in the hard X-ray regime, with different techniques, including amongst others: crystal interferometry, ${ }^{4-8}$ analyser crystal techniques ${ }^{9-15}$ free space propagation, ${ }^{16,17}$ grating interferometry ${ }^{18-20}$ and edge illumination. ${ }^{21,22}$ We focus on the latter for its capability to provide quantitative phase and (ultra-small-angle) scattering information, ${ }^{23,24}$ its compatibility with the polychromatic and divergent X-ray beam produced by conventional tubes ${ }^{25,26}$ and robustness of the set-up. ${ }^{27,28}$

E-mail: m.endrizzi@ucl.ac.uk 


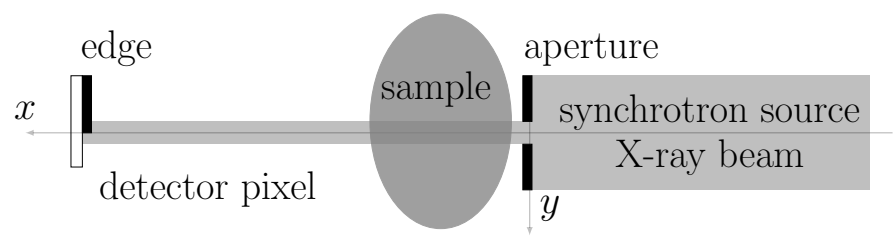

(a)

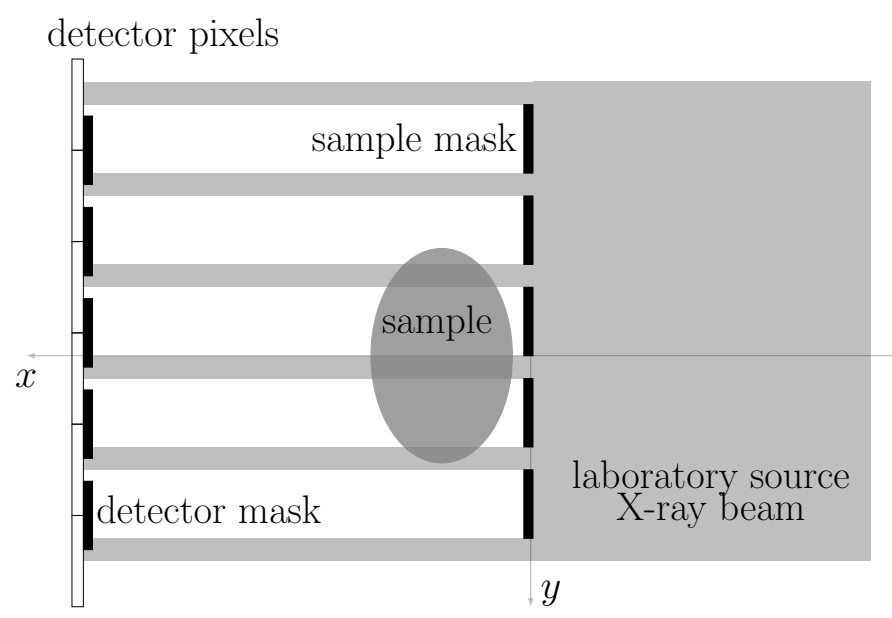

(b)

Figure 1: Typical edge illumination XPCI set-ups. (a) Synchrotron case where one single aperture is typically used and (b) laboratory where multiple apertures are simultaneously implemented through a mask enabling single shot area imaging. The illumination function describes how the detected intensity changes when one mask is scanned along $y$ with respect to the other. $y$ is also the direction along which the system is sensitive to refraction.

\section{METHODS}

The typical experimental set-up for an edge illumination XPCI experiment consists of an X-ray beam propagating from a source towards an aperture that is placed in close proximity of the sample. The function of this aperture is that of defining a narrow (few tens of micrometres) laminar beam that traverses the sample and then impinges on the edge of a second aperture that is placed before the detection system (see Figure 1a). In a synchrotron setting one aperture is typically used and the sample has to be scanned through the beam in order to acquire a two dimensional image. A set of apertures carved in a single structure to form a two dimensional mask, is usually preferred for laboratory settings to enable the acquisition of two-dimensional images in a single shot (see Figure 1b).

In a conventional edge illumination XPCI system, the intensity projections are acquired under complementary illumination conditions by physically moving one mask with respect to the other. The function that describes how the detected intensity changes, as a function of the relative displacement between the two masks, is called illumination function. It can be described as the convolution of two rectangular functions, representing the sample and detector apertures, and the source intensity distribution projected at the detector mask's plane. ${ }^{24,29}$ When one mask is shifted with respect to the other, the working point of the system moves along this illumination function.

For some applications, for example when system robustness and simplicity have to be maximised, the necessity to perform masks movements during data acquisition might be considered disadvantageous. For these cases, a system based on an asymmetric mask design ${ }^{30}$ can provide a solution where the imaging system is kept completely stationary during data collection and the only movement required is that of scanning the sample 
through. An asymmetric mask is obtained by modifying the conventional mask design into an asymmetric pattern of apertures in order to obtain complementary illumination conditions on adjacent pixels columns, rather than aiming at having the same illumination condition across the entire field of view. A three-way asymmetric mask, for example, is obtained by classifying the apertures into three groups such that they follow the pattern $\{1,2,3,1,2,3 \ldots 1,2,3\}$ along the $y$ direction. The positions of all the apertures belonging to group 1 remain unchanged. The positions of all the apertures belonging to group 2 are shifted by $-s$ and the positions of all the apertures belonging to group 3 are shifted by $+t$; with respect to the conventional uniformly spaced mask design. This can be extended to any desired number of groups where the relative shifts $s$ and $t$ can be tuned to obtain images at specific points along the illumination function.

A seven-way asymmetric mask was manufactured to the Authors' design by Creatv MicroTech, Inc. (Potomac, $\mathrm{MD})$. The X-ray absorbing material was gold electroplated onto a graphite substrate. Apertures were 23 and $29 \mu \mathrm{m}$ wide in the sample and detector mask respectively. While the detector mask's pitch was regular at 98 $\mu \mathrm{m}$, the sample apertures where relatively shifted by $( \pm 8, \pm 12, \pm 17) \mu \mathrm{m}$ with respect to the regular pitch of $79 \mu \mathrm{m}$ that would have harmonically matched the two masks via beam divergence. This was tested by using an edge illumination XPCI system based on a laboratory source (Molybdenum target rotating anode) operated at $45 \mathrm{kV} / 20 \mathrm{~mA}$. The sample and the detector masks were placed at 1.6 and $2 \mathrm{~m}$ from the source, respectively. The experimental results obtained with this asymmetric mask exhibit some characteristics that are related to the shape of the point spread function of the particular detector used. In this case the detector was a flat panel consisting of a CMOS sensor with directly deposited caesium iodide scintillator and a pixel size of $50 \mu \mathrm{m} \times$ $50 \mu \mathrm{m}$ (C9732DK-11, Hamamatsu, Japan). The illumination function measured experimentally were accurately reproduced by means of a numerical model that incorporates the actual detector characteristics. These results are then compared to those that would be expected by using a direct conversion detector. With the aim of extending the currently available field of view, a new prototype was designed. The sample mask was $15 \mathrm{~cm}$ high and $1.2 \mathrm{~cm}$ wide. Combined with the scanning of the sample, this enables a relatively large field of view to be achieved, up to $15 \mathrm{~cm} \times 50 \mathrm{~cm}$. The thickness of the absorbing septa was increased to $200 \mu \mathrm{m}$, such that the system could cope with the harder X-ray beams, $80-100 \mathrm{kVp}$. Each asymmetric group consists of four apertures, simultaneously providing four complementary illumination levels.

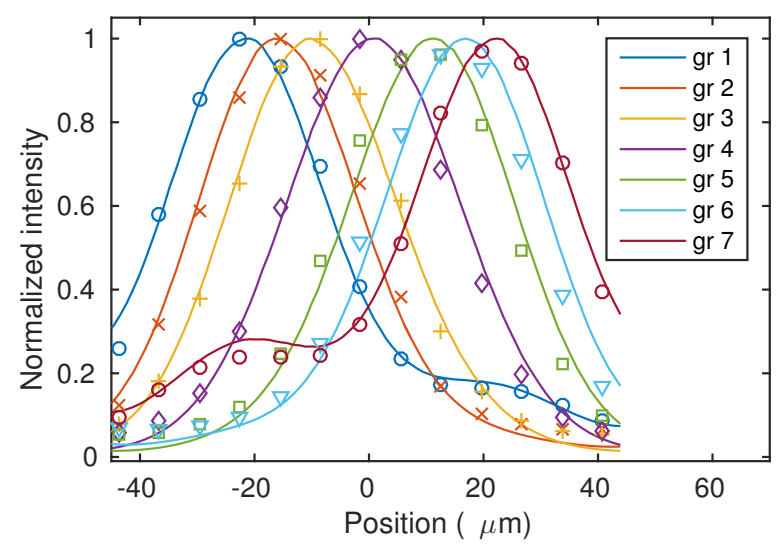

(a)

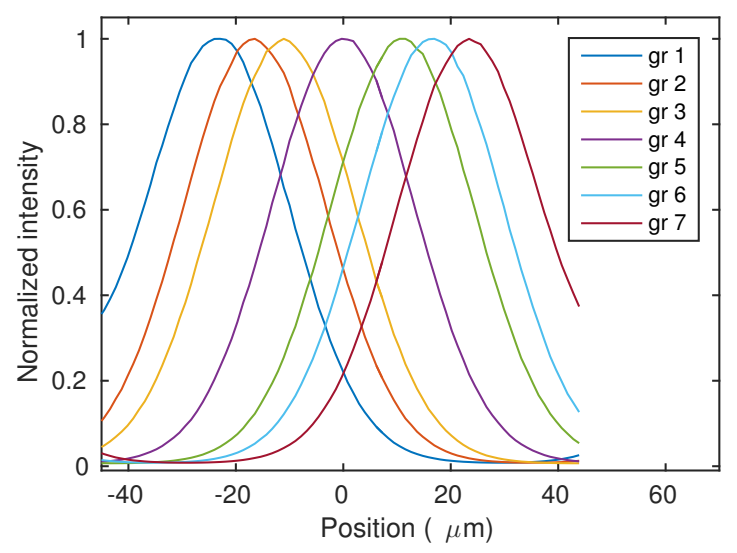

(b)

Figure 2: Experimental data and numerical simulation results for the seven-way asymmetric mask. (a) Experimental curves (markers) and numerical simulations (solid lines) that take into account the actual detector point spread function. A good agreement is obtained for the different illumination function measured with adjacent detector pixels columns (groups 1 to 7 ). The change in shape of the illumination functions is entirely related to the shape of the point spread function of the particular detector used in this case. (b) Numerical simulations assuming zero cross-talk between adjacent detector pixels columns: the illumination functions are only shifted and not distorted by the asymmetric design. 


\section{RESULTS}

The illumination functions experimentally measured with the seven-way asymmetric mask are reported in Figure 2a (markers). Adjacent detector columns result into laterally shifted illumination functions. This means that, for a given position of the sample mask (e.g. position $0 \mu \mathrm{m}$ ) seven different illumination fractions are simultaneously achieved in each column group. From the experimental data it is possible to observe that not only are the illumination functions shifted, but also changed in shape. This effect was numerically reproduced to a high level of accuracy, and it is plotted in Figure 2a (continuous lines). The numerical model used in this case is based on wave optics ${ }^{31}$ and accounted for the experimentally measured point spread function of the detector. ${ }^{32}$ The reason for this change in shape of the illumination functions is the non-negligible cross-talk between adjacent pixels resulting from the use of an indirect conversion detector, albeit used in line-skipping mode. ${ }^{33}$ This is confirmed by the numerical results of Figure $2 \mathrm{~b}$, where a detector with zero cross-talk was simulated. In this case, as a result of the asymmetric design, the illumination function are shifted one with respect to the other, but no distortion in their shape is observed. It has to be noted, however, that even in the case of the indirect conversion detector, the working point at position $0 \mu \mathrm{m}$ still provides the expected illumination fractions of $\pm 35 \%, \pm 60 \%, \pm 80 \%$ and $100 \%$ despite the distortion in the shape of the illumination functions. Experimental results obtained with the new prototype are shown in Figure 3. These illumination functions are simultaneously provided by four adjacent detector pixels columns, across the $15 \mathrm{~cm} \times 1.2 \mathrm{~cm}$ area covered by the sample mask. Data were collected at $100 \mathrm{kVp}$ and the complementary illumination fractions of approximately $100 \%, \pm 80 \%$ and $55 \%$ are simultaneously achieved.

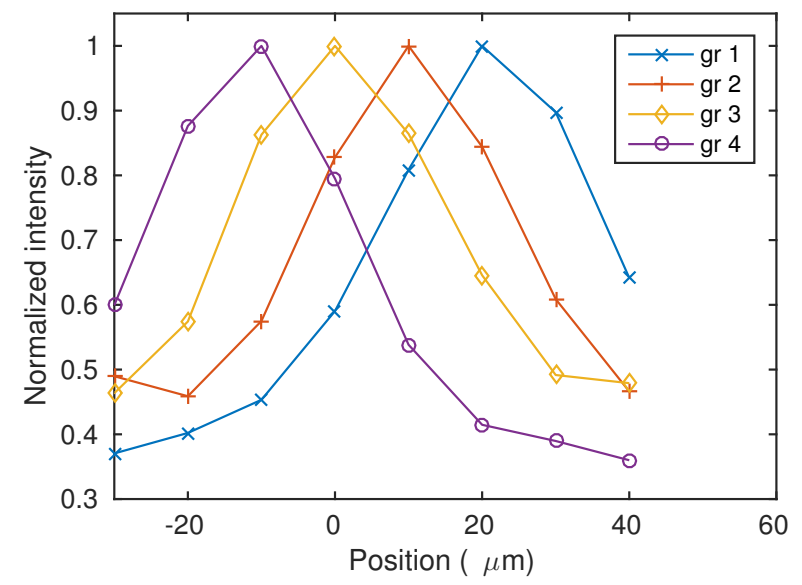

Figure 3: Experimental illumination functions of the four-way, large field-of-view system. Data were acquired at $100 \mathrm{kVp}$. Four adjacent detector columns simultaneously provide complementary illumination conditions at approximately $100 \%, \pm 80 \%$ and $55 \%$.

\section{CONCLUSION}

The concept of asymmetric masks for edge illumination X-ray phase-contrast imaging was presented and discussed. It enables the realisation of a system that is completely stationary during data acquisition, with the only requirement being that the object under investigation is scanned. For this reason this method is particularly suitable for those situations were the sample needs to be scanned through the imaging system, e.g. industrial inspection as well as some medical applications. A seven-way mask implementation of this concept was experimentally investigated and the result were accurately reproduced by numerical simulations accounting for the actual detector performance in terms of point spread function and pixel cross-talk. These concepts can be extended to a large field of view system capable of working at relatively high X-ray energies. Preliminary experimental results from the new scanner that is currently under development were presented. 


\section{ACKNOWLEDGMENTS}

This project was funded under the Innovative Research Call in Explosives and Weapons Detection 2013 initiative. This is a Cross-Government programme sponsored by a number of Departments and Agencies under the UK Governments CONTEST strategy in partnership with the US Department of Homeland Security, Science and Technology Directorate. Additional support to this work was provided by the UK Engineering and Physical Sciences Research Council Grant EP/I021884/1. ME was supported by the Royal Academy of Engineering under the RAEng Research Fellowships scheme.

\section{REFERENCES}

1. R. Fitzgerald, "Phase-sensitive x-ray imaging," Physics Today 53(7), pp. 23-26, 2000.

2. A. Bravin, P. Coan, and P. Suortti, "X-ray phase-contrast imaging: from pre-clinical applications towards clinics," Physics in Medicine and Biology 58(1), p. R1, 2013.

3. S. Wilkins, Y. I. Nesterets, T. Gureyev, S. Mayo, A. Pogany, and A. Stevenson, "On the evolution and relative merits of hard x-ray phase-contrast imaging methods," Philosophical Transactions of the Royal Society of London A: Mathematical, Physical and Engineering Sciences 372(2010), p. 20130021, 2014.

4. U. Bonse and M. Hart, "An x-ray interferometer," Applied Physics Letters 6, pp. 155 -156, 1965.

5. U. Bonse and M. Hart, "X-ray interferometer with long separated interfering beam paths," Applied Physics Letters 7, pp. $99-101,1965$.

6. M. Ando and S. Hosoya, An attempt at X-ray phase-contrast microscopy, University of Tokyo Press, 1971.

7. T. J. Davis, D. Gao, T. E. Gureyev, A. W. Stevenson, and S. W. Wilkins, "Phase-contrast imaging of weakly absorbing materials using hard X-rays," Nature 373, pp. 595-598, 1995.

8. A. Momose, T. Takeda, Y. Itai, and K. Hirano, "Phase-contrast x-ray computed tomography for observing biological soft tissues," Nature Medicine 2(4), pp. 473-475, 1996.

9. K. Goetz, E. Foerster, P. Zaumseil, M. P. Kalashnikov, I. A. Mikhailov, G. V. Sklizkov, and S. I. Fedotov, "Measurements of the parameters of shell targets for laser thermonuclear fusion using an X-ray schlieren method," Kvantovaia Elektronika Moscow 6, pp. 1037-1042, 1979.

10. E. Foerster, K. Goetz, and P. Zaumseil, "Double crystal diffractometry for the characterization of targets for laser fusion experiments," Kristall und Technik 15(8), pp. 937-945, 1980.

11. V. N. Ingal and E. A. Beliaevskaya, "X-ray plane-wave topography observation of the phase contrast from a non-crystalline object," Journal of Physics D Applied Physics 28, pp. 2314-2317, 1995.

12. D. Chapman, W. Thomlinson, R. E. Johnston, D. Washburn, E. Pisano, N. Gmür, Z. Zhong, R. Menk, F. Arfelli, and D. Sayers, "Diffraction enhanced x-ray imaging," Physics in Medicine and Biology 42, pp. 2015-2025, 1997.

13. L. Rigon, H.-J. Besch, F. Arfelli, R.-H. Menk, G. Heitner, and H. Plothow-Besch, "A new DEI algorithm capable of investigating sub-pixel structures," Journal of Physics D Applied Physics 36(26), pp. A260000A112, 2003.

14. E. Pagot, P. Cloetens, S. Fiedler, A. Bravin, P. Coan, J. Baruchel, J. Härtwig, and W. Thomlinson, "A method to extract quantitative information in analyzer-based x-ray phase contrast imaging," Applied Physics Letters 82, p. 3421, 2003.

15. M. N. Wernick, O. Wirjadi, D. Chapman, Z. Zhong, N. P. Galatsanos, Y. Yang, J. G. Brankov, O. Oltulu, M. A. Anastasio, and C. Muehleman, "Multiple-image radiography," Physics in Medicine and Biology 48, pp. 3875-3895, 2003.

16. A. Snigirev, I. Snigireva, V. Kohn, S. Kuznetsov, and I. Schelokov, "On the possibilities of x-ray phase contrast microimaging by coherent high-energy synchrotron radiation," Review of Scientific Instruments 66 (12), pp. 5486-5492, 1995.

17. S. W. Wilkins, T. E. Gureyev, D. Gao, A. Pogany, and A. W. Stevenson, "Phase-contrast imaging using polychromatic hard x-rays," Nature 384, pp. 335-338, 1996.

18. C. David, B. Nohammer, H. H. Solak, and E. Ziegler, "Differential x-ray phase contrast imaging using a shearing interferometer," Applied Physics Letters 81(17), pp. 3287-3289, 2002. 
19. T. Weitkamp, A. Diaz, C. David, F. Pfeiffer, M. Stampanoni, P. Cloetens, and E. Ziegler, "X-ray phase imaging with a grating interferometer," Optics Express 13, pp. 6296-6304, Aug 2005.

20. F. Pfeiffer, T. Weitkamp, O. Bunk, and C. David, "Phase retrieval and differential phase-contrast imaging with low-brilliance X-ray sources," Nature Physics 2, pp. 258-261, 2006.

21. A. Olivo, F. Arfelli, G. Cantatore, R. Longo, R. H. Menk, S. Pani, M. Prest, P. Poropat, L. Rigon, G. Tromba, E. Vallazza, and E. Castelli, "An innovative digital imaging set-up allowing a low-dose approach to phase contrast applications in the medical field," Medical Physics 28, pp. 1610-1619, 2001.

22. A. Olivo and R. Speller, "A coded-aperture technique allowing x-ray phase contrast imaging with conventional sources," Applied Physics Letters 91(7), p. 074106, 2007.

23. P. R. Munro, K. Ignatyev, R. D. Speller, and A. Olivo, "Phase and absorption retrieval using incoherent X-ray sources," Proc. Natl. Acad. Sci. USA 109(35), pp. 13922-13927, 2012.

24. M. Endrizzi, P. C. Diemoz, T. P. Millard, J. L. Jones, R. D. Speller, I. K. Robinson, and A. Olivo, "Hard x-ray dark-field imaging with incoherent sample illumination," Applied Physics Letters 104(2), p. 024106, 2014.

25. P. R. T. Munro and A. Olivo, "X-ray phase-contrast imaging with polychromatic sources and the concept of effective energy," Phys. Rev. A 87, p. 053838, 2013.

26. M. Endrizzi, F. A. Vittoria, G. Kallon, D. Basta, P. C. Diemoz, A. Vincenzi, P. Delogu, R. Bellazzini, and A. Olivo, "Achromatic approach to phase-based multi-modal imaging with conventional x-ray sources," Optics Express 23(12), pp. 16473-16480, 2015.

27. T. P. Millard, M. Endrizzi, K. Ignatyev, C. K. Hagen, P. R. T. Munro, R. D. Speller, and A. Olivo, "Method for automatization of the alignment of a laboratory based x-ray phase contrast edge illumination system," Review of Scientific Instruments 84(8), p. 083702, 2013.

28. M. Endrizzi, D. Basta, and A. Olivo, "Laboratory-based x-ray phase-contrast imaging with misaligned optical elements," Applied Physics Letters 107(12), p. 124103, 2015.

29. M. Endrizzi and A. Olivo, "Absorption, refraction and scattering retrieval with an edge-illumination-based imaging setup," Journal of Physics D: Applied Physics 47(50), p. 505102, 2014.

30. M. Endrizzi, A. Astolfo, F. A. Vittoria, T. P. Millard, and A. Olivo, "Asymmetric masks for laboratory-based x-ray phase-contrast imaging with edge illumination," Scientific reports 6, 2016.

31. F. A. Vittoria, P. C. Diemoz, M. Endrizzi, L. Rigon, F. C. Lopez, D. Dreossi, P. R. T. Munro, and A. Olivo, "Strategies for efficient and fast wave optics simulation of coded-aperture and other x-ray phase-contrast imaging methods," Appl. Opt. 52(28), pp. 6940-6947, 2013.

32. M. Endrizzi, P. Oliva, B. Golosio, and P. Delogu, "Cmos aps detector characterization for quantitative x-ray imaging," Nucl. Instr. Meth. Phys. Res. A 703(0), pp. $26-32,2013$.

33. K. Ignatyev, P. R. T. Munro, R. D. Speller, and A. Olivo, "Effects of signal diffusion on x-ray phase contrast images," Review of Scientific Instruments 82(7), p. 073702, 2011. 